\title{
The Effect of Earth's Magnetic Field on the HF Radio Wave Modes at the Heated Subionosphere
}

\author{
M. CANYILmaz ${ }^{a, *}$, R. Atici ${ }^{b}$ And E. GuZel ${ }^{a}$ \\ ${ }^{a}$ Department of Physics, Faculty of Sciences, Frrat University, 23119 Elazig, Turkey \\ ${ }^{b}$ Department of Physics, Faculty of Arts and Sciences, Mus Alparslan University, 23119 Mus, Turkey
}

(Received September 19, 2012)

\begin{abstract}
In this study, the effect of the actual geometry of the magnetic field on the electron temperature has been investigated daily and seasonally for the electromagnetic wave travelling at subionosphere. The electron energy balance equation has been obtained by using the ideal gas approximation. The results show that there are notable differences between the two situations ( $B\|k\| z$ and $B$ real, $\| z$ ). According to our theoretical study, the maximum changes are shown at L- and R-mode, small effect on X-mode and no variation on O-mode due to the real geometry of the magnetic field. Besides, the heights of the maximum points are changeable daily and seasonally for all modes at the subionosphere.
\end{abstract}

DOI: 10.12693 /APhysPolA.123.786

PACS: 94.20.Bb, 94.20.ws, 94.20.wj, 41.20.Jb, 84.40.-x

\section{Introduction}

The highest frequency waves reflected from the ionosphere are high-frequency (HF) waves which cover the range of $3-30 \mathrm{MHz}$. The properties of the ionosphere have been investigated by examining factors affecting the propagation and reflection of the radio waves in this HF band reflected from the ionosphere [1].

The HF radio wave is exposed to many physical events in the ionosphere until it reaches the receiver by reflecting from the ionosphere after sending from the transmitter. The most important one of these is absorption. The HF radio wave passing through the ionosphere transfers the electrons and ions in free state within weakly ionized plasma as a part of its energy. The transferred energy increases the average kinetic energy of electrons and ions in free state. If the electrons and ions with increased energy do not collide with low-energy neutral particles, then a large part of lost energy of the radio wave is converted to the electromagnetic energy and continues to the propagation too much unchanged intensity of the wave. However, if the electrons and ions collide with neutral particles, then a large part of this energy is lost and the wave energy is absorbed [2-5].

High-powerful radio waves increase the temperature and change the electron and ion densities through the collisions of the electrons with the ions and with the neutral molecules and atoms in the perturbed region of the ionosphere. This alters the high-powerful radio waves absorption. Thus, the heating of the ionosphere in the field of high-powerful radio waves should excite new plasma oscillations and enhance those already present there, and give rise to formation of an oscillating tur-

*corresponding author; e-mail: mcanyilmaz@firat.edu.tr bulized region. This strongly influences the conditions of radio wave propagation in the perturbed region of plasma, causing them to become more strongly absorbed and scattered [6].

The above-mentioned effects are only a few of the nonlinear effects occurring in the ionospheric plasma. Nonlinear effects were observed by Gurevich et al. [7, 8]. They were described as effects of the self-action of a high power radio wave. These effects lead to a sharp increase of the wave absorption under certain conditions and decrease of absorption (plasma "translucence" effect) under others [9].

In the 1980s the large number of theoretical and experimental studies on artificially heated D-region electrons with the HF radio waves have been started to use high-power transmitters in Norway, Sweden and Finland. The most important one of the experimental studies is European Incoherent Scatter Scientific Association (EISCAT) receiver in Norway, in Tromso. In addition, located in Arecibo in Puerto Rico, and in Alaska, the High-frequency Active Auroral Research Program (HAARP) and High-Power Auroral Stimulation (HIPAS) transmitters are also available. These experimental studies not only lead to deeper comprehension on the microscopic and macroscopic, long-term and short-term, and linear and nonlinear processes caused by the HF heating of the ionosphere but also make the comparison of theory, experiment and simulation available. Theoretical analysis is made by means of electron energy balance equation using an ideal gas approximation of the electron gas [10-15].

In this theoretical research, the effect of a high-power HF radio wave which spreads as vertical on the electron temperature in the subionosphere depending on the actual geometry of the magnetic field of the Earth has been studied. These effects are investigated as daily and seasonal in terms of different wave modes. 


\section{Theoretical background}

The force acting on the electron is

$$
m \frac{\mathrm{d} \boldsymbol{v}}{\mathrm{d} t}=-e(\boldsymbol{E}+\boldsymbol{v} \times \boldsymbol{B})-m \nu v \boldsymbol{v},
$$

where $\boldsymbol{E}$ is electrical field, $\boldsymbol{B}$ is magnetic field, $\boldsymbol{v}$ and $m$ are the velocity and mass of electron, respectively. $\nu$ is total collisions frequency and sum of the electron-neutral and electron-ion collision frequencies $[16,17]$ :

$$
\nu_{\mathrm{e}}=\nu_{\mathrm{en}}+\nu_{\mathrm{ei}},
$$

in which

$$
\begin{aligned}
& \nu_{\mathrm{en}}=2.33 \times 10^{-17}\left[\mathrm{~N}_{2}\right]\left(1-1.21 \times 10^{-4} T_{\mathrm{e}}\right) T_{\mathrm{e}} \\
& \quad+1.8 \times 10^{-16}\left[\mathrm{O}_{2}\right]\left(1+3.6 \times 10^{-2} \sqrt{T}_{\mathrm{e}}\right) \sqrt{T}_{\mathrm{e}} \\
& +8.9 \times 10^{-16}[\mathrm{O}]\left(1+5.7 \times 10^{-4} T_{\mathrm{e}}\right) \sqrt{T}_{\mathrm{e}}
\end{aligned}
$$

and

$$
\nu_{\mathrm{ei}}=N_{\mathrm{e}} 10^{-6}\left[59+4.18 \log \left(\frac{T_{\mathrm{e}}^{3}}{N_{\mathrm{e}}}\right)\right] T_{\mathrm{e}}^{-3 / 2},
$$

where $T_{\mathrm{e}},\left[\mathrm{N}_{2}\right],\left[\mathrm{O}_{2}\right],[\mathrm{O}]$ and $N_{\mathrm{e}}$ are electron temperature in $\mathrm{K}$, densities of nitrogen molecule, oxygen molecule, oxygen atom in $\mathrm{m}^{-3}$, respectively.

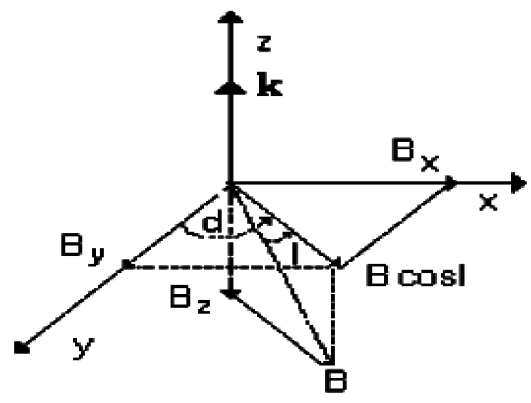

Fig. 1. Geometry of Earth's magnetic field $(\boldsymbol{B})$ and $k[18]$.

The geometry of the geomagnetic field in the Northern hemisphere is given in Fig. 1. The $z$-axis of the coordinate system with its origin located on the ground is vertical upwards. The $x$ - and $y$-axis are geographic eastward and northward in the Northern hemisphere, respectively.

The geomagnetic field is $\boldsymbol{B}=B_{x} \boldsymbol{a}_{x}+B_{y} \boldsymbol{a}_{y}+B_{z} \boldsymbol{a}_{z}$ with components $B_{x}=B \cos I \sin d, B_{y}=B \cos I \cos d$ and $B_{z}=-B \sin I$. Also $I$ and $d$ are the magnetic dip and declination angles. In a Cartesian coordinate system, the solution of Eq. (1) can be written as

$$
\boldsymbol{J}=\sigma \boldsymbol{E},
$$

where $\boldsymbol{J}$ is the current density and $\sigma$ is the ionospheric conductivity tensor. From Maxwell's equations the following wave equation can be obtained [16]:

$$
n^{2} \boldsymbol{E}-\boldsymbol{n}(\boldsymbol{n} \cdot \boldsymbol{E})-\left[I+\frac{\mathrm{i} \sigma}{\varepsilon_{0} \omega}\right] \boldsymbol{E}=0
$$

in which $I$ is unit matrix and $n$ is the refractive index. By using the geometry and solving Eq. (4), we can obtain the basic dispersion relation as follows:

$$
\operatorname{det}(\boldsymbol{M})=0 .
$$

Thus, different modes of the HF wave are obtained by solving basic dispersion relation and the refractive index $n$ can be obtained in the terms of plasma parameters. The HF wave is travelling in the $z$-direction in the ionosphere. The solution of Eq. (5) gives two modes. These are plasma oscillations and polarization waves ( $\mathrm{P}$-waves). Polarized waves are divided into two as the right (right-handed, R) and left (left-handed, L):

$$
\begin{aligned}
n_{\mathrm{R}}^{2} & =1-\frac{X(1-Y \sin I)}{(1-Y \sin I)^{2}+Z^{2}} \\
& +\mathrm{i} \frac{X Z}{(1-Y \sin I)^{2}+Z^{2}}, \\
n_{\mathrm{L}}^{2} & =1-\frac{X(1+Y \sin I)}{(1+Y \sin I)^{2}+Z^{2}} \\
& +\mathrm{i} \frac{X Z}{(1+Y \sin I)^{2}+Z^{2}} .
\end{aligned}
$$

Despite of the HF wave is propagating in the $z$-direction, there are also waves travelling in the $y$ - (or $x-$ ) direction due to the geometry of the geomagnetic field. These are ordinary (O-wave) and extraordinary (X-wave) waves [16]:

$$
\begin{aligned}
n_{0}^{2} & =1-\frac{X}{1+Z^{2}}+\mathrm{i} \frac{X Z}{1+Z^{2}}, \\
n_{x}^{2} & =1-X\left[(1-X)\left(1-X-Y^{2} \cos ^{2} I \cos ^{2} d\right)\right. \\
& \left.+Z^{2}\right] /\left[\left(1-X-Z^{2}-Y^{2} \cos ^{2} I \cos ^{2} d\right)^{2}\right. \\
& \left.+Z^{2}(2-X)^{2}\right]+\mathrm{i} Z X\left[\left[(1-X)^{2}+Z^{2}\right.\right. \\
& \left.+Y^{2} \cos ^{2} I \cos ^{2} d\right] /\left(1-X-Z^{2}\right. \\
& \left.\left.-Y^{2} \cos ^{2} I \cos ^{2} d\right)^{2}+Z^{2}(2-X)^{2}\right],
\end{aligned}
$$

where $X, Y$, and $Z$ are $X=\frac{\omega_{\mathrm{p}}^{2}}{\omega^{2}}, Y=\frac{\omega_{\mathrm{ce}}}{\omega}$, and $Z=\frac{v_{\mathrm{e}}}{\omega}$.

These equations show that the refractive index has a real and a complex component as $n^{2}=(\mu+\mathrm{i} \chi)^{2}=$ $M+$ i $N$. The complex component defines the characteristics of the absorption, while real component defines properties of dispersion and breaking of the media. The absorption part transformed into heat and electromagnetic noise of the radio wave energy through interactions between ionospheric electrons and neutral atmosphere. Most of the absorption occurs in the ionospheric D-region which reaches the maximum of the electron density production and electron collision frequency. In this case, the complex component of the refractive index is given by

$$
\chi^{2}=\frac{1}{2}\left[\left(M^{2}+N^{2}\right)^{1 / 2}-M\right] .
$$

Using the ideal gas approach to electron gas, the electron temperature change based on the absorption is defined by the following nonlinear differential Eq. (9): 


$$
\frac{\mathrm{d} T_{\mathrm{e}}}{\mathrm{d} t}=\frac{2}{3 k_{\mathrm{B}} N_{\mathrm{e}}}\left(-\chi \frac{2 \omega S}{c}-L\right),
$$

in which $k_{\mathrm{B}}$ is the Boltzmann constant, $S$ is the energy flux of the HF radio wave and $L$ defines sum of the energy losses as a result of the electron collision processes. The energy flux is determined as

$$
S(z)=\frac{\mathrm{ERP}}{4 \pi z^{2}} \exp \left(-2 \frac{\omega}{c} \int_{0}^{z} \chi\left(z^{\prime}\right) \mathrm{d} z^{\prime}\right),
$$

where ERP is the effective radiation power of the transmitter $[2,10]$.

Also, $L$ values are taken as follows [19]:

$$
\begin{aligned}
& L_{\text {elast }}\left(\mathrm{e}, \mathrm{N}_{2}\right)=1.89 \times 10^{-44} N_{\mathrm{e}} N_{\mathrm{N}_{2}} \\
& \times\left(1-1.21 \times 10^{-4} T_{\mathrm{e}}\right) T_{\mathrm{e}}\left(T_{\mathrm{e}}-T_{\mathrm{n}}\right), \\
& L_{\text {elast }}\left(\mathrm{e}, \mathrm{O}_{2}\right)=1.29 \times 10^{-43} N_{\mathrm{e}} N_{\mathrm{O}_{2}} \\
& \times\left(1+3.6 \times 10^{-2} T_{\mathrm{e}}^{\frac{1}{2}}\right) T_{\mathrm{e}}^{\frac{1}{2}}\left(T_{\mathrm{e}}-T_{\mathrm{n}}\right), \\
& L_{\text {rot }}\left(\mathrm{e}, \mathrm{N}_{2}\right)=4.65 \times 10^{-39} N_{\mathrm{e}} N_{\mathrm{N}_{2}}\left(\frac{T_{\mathrm{e}}-T_{\mathrm{n}}}{T_{\mathrm{e}}^{\frac{1}{2}}}\right) \text {, } \\
& L_{\mathrm{rot}}\left(\mathrm{e}, \mathrm{O}_{2}\right)=1.11 \times 10^{-38} N_{\mathrm{e}} N_{\mathrm{O}_{2}}\left(\frac{T_{\mathrm{e}}-T_{\mathrm{n}}}{T_{\mathrm{e}}^{\frac{1}{2}}}\right) \text {, } \\
& L_{\mathrm{vib}}\left(\mathrm{e}, \mathrm{N}_{2}\right)=4.79 \times 10^{-37} N_{\mathrm{e}} N_{\mathrm{N}_{2}} \exp \left(f_{1} \frac{T_{\mathrm{e}}-2000}{2000 T_{\mathrm{e}}}\right) \\
& \times\left[\exp \left(-g \frac{T_{\mathrm{e}}-T_{\mathrm{n}}}{T_{\mathrm{e}} T_{\mathrm{n}}}\right)-1\right], \\
& f_{1}=1.06 \times 10^{4}+7.51 \times 10^{3} \\
& \times \tanh \left(11 \times 10^{-4}\left(T_{\mathrm{e}}-1800\right)\right), \\
& g=3300+1.233\left(T_{\mathrm{e}}-1000\right) \\
& -2.056 \times 10^{-4}\left(T_{\mathrm{e}}-1000\right)\left(T_{\mathrm{e}}-4000\right), \\
& L_{\text {vib }}\left(\mathrm{e}, \mathrm{O}_{2}\right)=8.32 \times 10^{-38} N_{\mathrm{e}} N_{\mathrm{NO}_{2}} \exp \left(f_{2} \frac{T_{\mathrm{e}}-700}{700 T_{\mathrm{e}}}\right) \\
& \times\left[\exp \left(-2770 \frac{T_{\mathrm{e}}-T_{\mathrm{n}}}{T_{\mathrm{e}} T_{\mathrm{n}}}\right)-1\right], \\
& f_{2}=3300-839 \sin \left(1.91 \times 10^{-5}\left(T_{\mathrm{e}}-2700\right)\right) \text {. }
\end{aligned}
$$

\section{The calculation algorithm}

Typically, the electron temperature in the ionosphere responds rapidly (a few seconds) to changing conditions and, therefore, the electron temperature is generally in a quasi-steady state $(\partial / \partial t \rightarrow 0)$. Furthermore, at low altitudes, thermal conduction is not important because the neutrals are effective in inhibiting the flow of heat. Under these circumstances, the electron temperature is determined by a balance between local heating and cooling processes [20]. In the equilibrium case is $\frac{\mathrm{d} T_{\mathrm{e}}}{\mathrm{d} t}=0$ and Eq. (11) can be solved as a function of $T_{\mathrm{e}}$ [10]. The needed parameters for this calculation are $\omega, N_{\mathrm{e}}, B,\left[\mathrm{~N}_{2}\right]$, $\left[\mathrm{O}_{2}\right],[\mathrm{O}]$ and $T_{\mathrm{n}}$. In this study, the data of electron den- sity were taken from the IRI-2007 model; neutral atmosphere parameters were taken from MSISE-90 model [20].

In the equilibrium case, the electron temperature in a desired height is calculated with the equalization to zero of the difference between energy transfer and loss to the media. The integral in Eq. (12) was calculated with steps of 100 meters according to the trapezoidal method and the roots of Eq. (12) were found by the bisection method.

\section{Results}

To investigate the effect of the HF radio wave of the electron temperature at the subionosphere, appropriate values are selected to the EISCAT transmitter such as $f=2.8 \mathrm{MHz}$ and ERP $=200 \mathrm{MW}$. Magnetic field has taken as $B_{0}=4 \times 10^{-5} \mathrm{~T}$. All parameters are calculated for the $39^{\circ} 41^{\prime} \mathrm{N}, 38^{\circ} 14^{\prime} \mathrm{E}$ and $I=55^{\circ}, d=3^{\circ}$. Year, day and time are taken as 2009, equinox, local time, respectively.

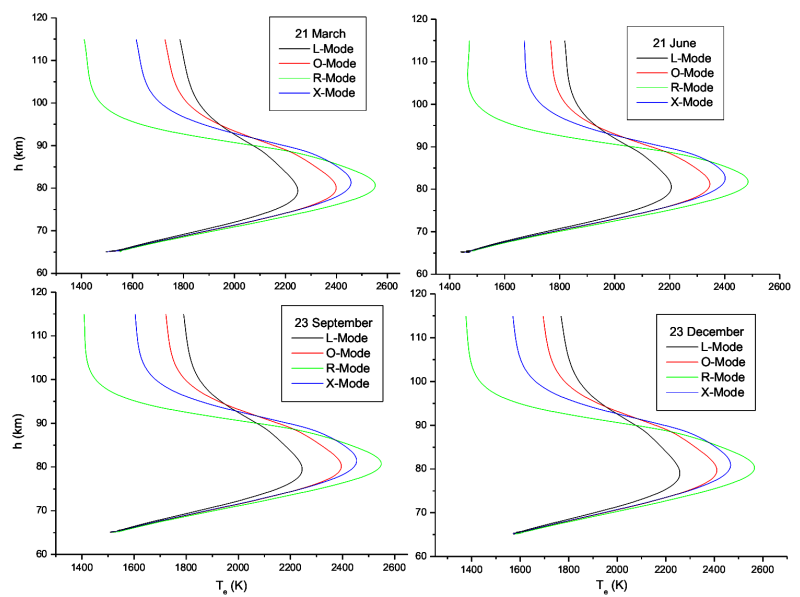

Fig. 2. The effect of the different modes of the HF radio wave over the electron temperature for the sunrise times.

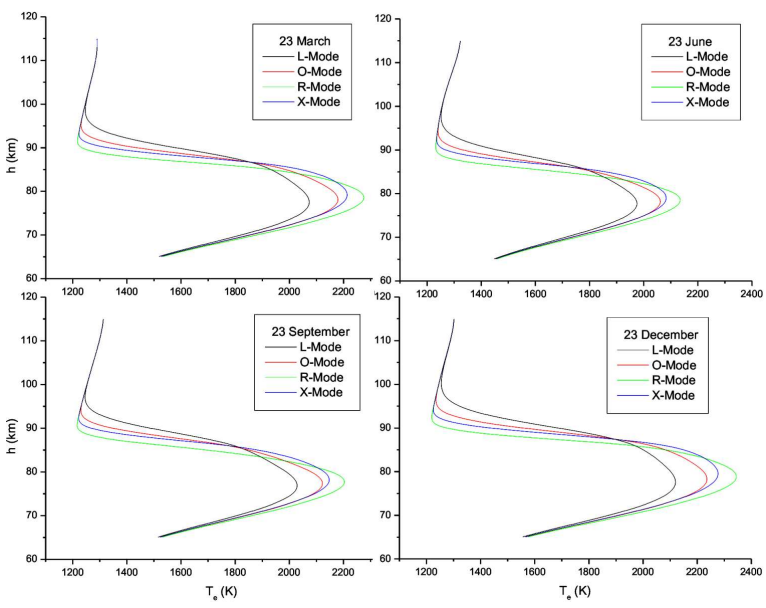

Fig. 3. The effect of the different modes of the HF radio wave over the electron temperature for the $14.00 \mathrm{LT}$. 
The effect of the different modes of the HF radio wave over the electron temperature for the sunrise and 14.00 LT is given in Fig. 2 and Fig. 3. The sunrise times are 05.17, 03.52, 05.00 and 06.34 for 21 March, 21 June, 23 September and 21 December, respectively. HF radio wave and magnetic field is at the $z$-direction.

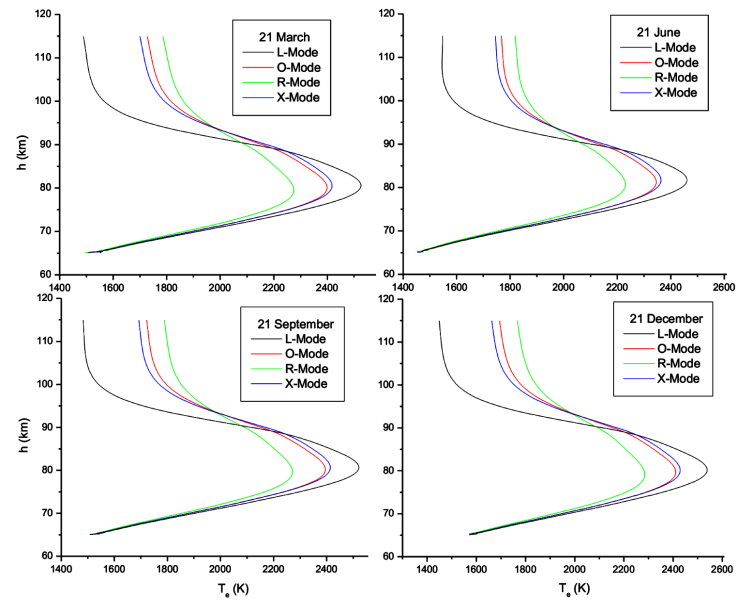

Fig. 4. The effect of the different modes of the HF radio wave over the electron temperature for the sunrise (magnetic field real geometry).

The maximum and minimum effects are seen at the R-mode and L-mode for the sunrise and 14.00 LT, respectively. The electron temperature is bigger than $2400 \mathrm{~K}$ for $\mathrm{R}$ - and $\mathrm{X}$-mode at the sunrise. But, the electron temperature decreases according to the sunrise at the af- ternoon. The maximum and minimum effects are at the same modes.

The variations of the electron temperature of the different modes are given in Fig. 4 and Fig. 5. HF radio wave is at the $z$-direction and magnetic field's real geometry is used at the calculations.

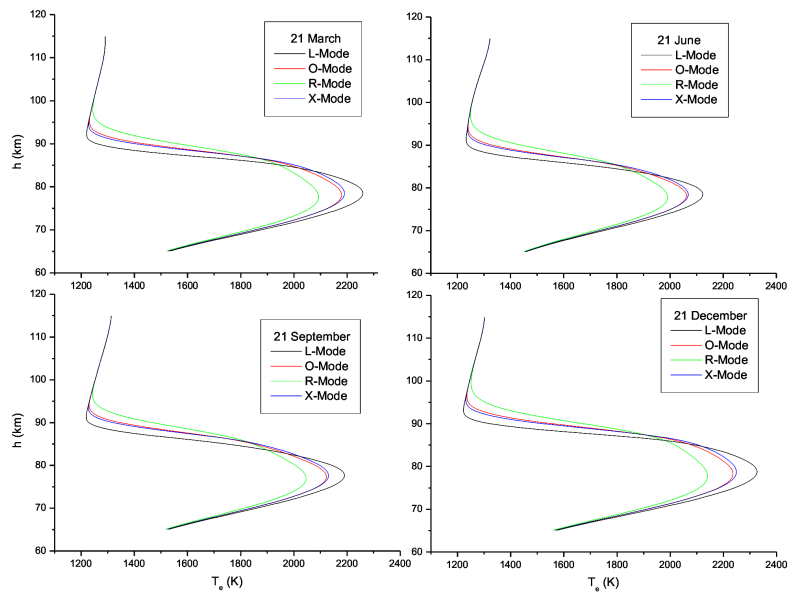

Fig. 5. The effect of the different modes of the HF radio wave over the electron temperature for the $14.00 \mathrm{LT}$ (magnetic field real geometry).

The maximum and minimum effects are opposite when the magnetic field real geometry is used. R- and L-mode are exchanged. The maximum values of electron temperature with height are given at Table.

The maximum values of electron temperature and height.

TABLE

\begin{tabular}{|c|c|c|c|c|c|c|c|c|c|}
\hline & & \multicolumn{4}{|c|}{ Electron temperature [K] } & \multicolumn{4}{|c|}{ Height $[\mathrm{km}]$} \\
\hline & & 21 March & 21 June & 23 Sept. & 21 Dec. & 21 March & 21 June & 23 Sept. & 21 Dec. \\
\hline \multirow{4}{*}{$\begin{array}{c}\text { sunrise } \\
(B\|k\| z)\end{array}$} & R-mode & 2552.6 & 2484.7 & 2548.2 & 2565.6 & 80.7 & 81.8 & 80.7 & 80.3 \\
\hline & L-mode & 2248.1 & 2205.8 & 2244.7 & 2257.8 & 79.4 & 80.5 & 79.5 & 79 \\
\hline & O-mode & 2399.1 & 2345.8 & 2395.1 & 2410.4 & 80.1 & 81.2 & 80.2 & 79.7 \\
\hline & $\mathrm{X}$-mode & 2457.2 & 2401.6 & 2453.7 & 2467.2 & 81.3 & 82.7 & 81.4 & 81 \\
\hline \multirow{4}{*}{$\begin{array}{c}14.00 \mathrm{LT} \\
(B\|k\| z)\end{array}$} & R-mode & 2274.8 & 2133.8 & 2203.5 & 2344.7 & 78.5 & 78.2 & 77.7 & 78.9 \\
\hline & L-mode & 2073.4 & 1974.9 & 2028.9 & 2119.7 & 77.5 & 77.5 & 76.7 & 77.5 \\
\hline & O-mode & 2179.3 & 2061.4 & 2122.6 & 2235.1 & 78.1 & 78.1 & 77.3 & 78.3 \\
\hline & X-mode & 2213.3 & 2083.1 & 2147.4 & 2276.9 & 79.2 & 78.9 & 78.2 & 79.6 \\
\hline \multirow{4}{*}{$\begin{array}{c}\text { sunrise } \\
(k \| z) \\
(B \text { real })\end{array}$} & R-mode & 2274.7 & 2230.7 & 2271.2 & 2284.7 & 79.5 & 80.7 & 79.6 & 79.1 \\
\hline & L-mode & 2525.7 & 2460.6 & 2521.4 & 2538.4 & 80.5 & 81.7 & 80.7 & 80.2 \\
\hline & O-mode & 2399.1 & 2345.8 & 2395.1 & 2410.4 & 80.1 & 81.2 & 80.2 & 79.7 \\
\hline & $\mathrm{X}$-mode & 2417.8 & 2363.3 & 2413.9 & 2428.7 & 80.5 & 81.7 & 80.7 & 80.1 \\
\hline \multirow{4}{*}{$\begin{array}{c}14.00 \mathrm{LT} \\
(k \| z) \\
(B \text { real })\end{array}$} & R-mode & 2092.7 & 1991.3 & 2046.3 & 2140.7 & 77.6 & 77.8 & 76.9 & 77.7 \\
\hline & L-mode & 2259.1 & 2123.6 & 2190.6 & 2326.5 & 78.5 & 78.4 & 77.6 & 78.8 \\
\hline & O-mode & 2179.3 & 2061.4 & 2122.6 & 2235.1 & 78.1 & 78.1 & 77.3 & 78.3 \\
\hline & $\mathrm{X}$-mode & 2190.1 & 2068.4 & 2130.7 & 2249 & 78.5 & 78.4 & 77.6 & 78.7 \\
\hline
\end{tabular}




\section{Conclusion}

The ionosphere can be heated by powerful HF radio wave. The effect of the actual geometry of the magnetic field on this heating has been investigated. In our theoretical calculations a difference was found between $B \| z$ and $B$-real geometry. The collision frequency and electron temperature $\left(v_{\mathrm{e}} \sim T_{\mathrm{e}}\right)$ are high at the subionosphere.

Figures $2-5$ are showing the effect of the different modes of the $\mathrm{HF}$ radio wave over the electron temperature for the sunrise, $14.00 \mathrm{LT}$ and equinox days. The maximum and minimum effects are at $\mathrm{R}$ - and L-mode, respectively, where the magnetic field is at the $z$-direction in Figs. 2 and 3. When we add the real geometry of the magnetic field to our calculations, an opposite condition occurs between these two modes as shown in Fig. 4 and 5 .

The electron temperature decreases about $40 \mathrm{~K}$ at $\mathrm{X}$-mode between these two conditions. But, there is not any change at $\mathrm{O}$-mode because of the non-effect of the magnetic field (Eq. (8)).

The maximum values of electron temperature and height for equinox days and different hours are given in Table. Sunrise time electron temperature is nearly 200$300 \mathrm{~K}$ bigger than $14.00 \mathrm{LT}$ for all modes, days. Also, the absorption height is decreasing with increase of the electron density. The energy in the ionosphere is shared between the existing electrons. The average kinetic energy of electrons is decreasing with the increase of the electron density. So that, high-power radio wave energy is absorbed at a lower height.

\section{References}

[1] M. Aydogdu, E. Guzel, A. Yesil, O. Ozcan, M. Canyilmaz, Nuovo Cimento Della Societa Italiana Di Fisica C-Geophysics and Space Physics 30, 243 (2007).

[2] M. Rietveld, H. Kopka, P. Stubbe, J. Atmospheric and Terrestr. Phys. 48, 311 (1986).
[3] E. Belova, A. Pashin, W. Lyatsky, J. Atmospheric and Terrestr. Phys. 57, 265 (1995).

[4] Y. Taranenko, U. Inan, T. Bell, Geophys. Res. Lett. 19, 61 (1992).

[5] R. Barr, P. Stubbe, Geophys. Res. Lett. 18, 1035 (1991).

[6] A.V. Gurevich, Nonlinear Phenomena in the Ionosphere, Vol. 10, Springer Verlag, New York 1978.

[7] A. Gurevich, A. Lukyanov, K. Zybin, Phys. Lett. A 211, 363 (1996).

[8] A.V. Gurevich, T. Hagfors, H. Carlson, A.V. Lukyanov, K.P. Zybin, Phys. Lett. A 246, 335 (1998).

[9] A. Gurevich, H. Carlson, M. Kelley, T. Hagfors, A. Karashtin, K. Zybin, Phys. Lett. A 251, 311 (1999).

[10] A. Kero, T. Bösinger, P. Pollari, E. Turunen, M. Rietveld, Ann. Geophys. 18, 1210 (2000).

[11] F. He, Z. Zhao, Frontiers of Electrical and Electronic Engineering in China 4, 205 (2009).

[12] T. Robinson, Phys. Rep. 179, 79 (1989).

[13] M. Rietveld, H. Kohl, H. Kopka, P. Stubbe, J. Atmospheric and Terrestr. Phys. 55, 577 (1993).

[14] T. Jones, T. Robinson, P. Stubbe, H. Kopka, J. Atmospheric and Terrestr. Phys. 48, 1027 (1986).

[15] T. Borisova, N. Blagoveshchenskaya, V. Kornienko, M. Rietveld, B. Thidé, T. Leyser, Geomagn. Aeron. 45, 367 (2005).

[16] M. Aydogdu, A. Yesil, E. Güzel, J. Atmospheric and Solar-Terrestr. Phys. 66, 343 (2004).

[17] R.W. Schunk, A.F. Nagy, Ionospheres: Physics, Plasma Physics, and Chemistry, Cambridge Univesity Press, Cambridge 2004.

[18] M. Aydogdu, O. Ozcan, J. Electromagnetic Waves and Applications 14, 1289 (2000).

[19] J.V. Rodriguez, Ph.D. Thesis, Stanford University, California 1994.

[20] A.E. Hedin, J. Geophys. Res. 96, 1159 (1991). 